\title{
LA EMOCIÓN COMO FUNDAMENTO DE LA CLASE: MOVIMIENTO OBRERO Y SOCIALISMO EN LA VIZCAYA FINISECULAR*
}

\author{
EMOTION AS THE FOUNDATION OF CLASS: WORKING CLASS \\ MOVEMENT AND SOCIALISM IN FIN-DE-SIECLE BISCAY
}

\author{
EMOÇÃO COMO FUNDAMENTO DA CLASSE: MOVIMENTO OPERÁRIO E \\ SOCIALISMO NA VIZCAYA DE FINAIS DO SÉCULO XIX
}

\author{
SARA HIDALGO GARCÍA DE ORELLÁN** \\ Universidad del País Vasco/Euskal Herriko Unibertsitatea \\ https://doi.org/10.46553/EHE.23.1.2021.p39-54
}

\begin{abstract}
Resumen
Este trabajo propone un estudio renovado del proceso de formación de la conciencia de clase en la Vizcaya industrial de finales del XIX, centrándonos en el movimiento socialista, y usando el andamiaje teórico y metodológico de la historia de las emociones. Se propone analizar la conciencia de clase a través del estudio de las expresiones emocionales, prácticas y rituales que consideramos que constituyen lo que llamamos régimen emocional socialista rojo, basamento emocional del programa político socialista en esta zona. Este régimen se definió por el pacifismo en los actos reivindicativos; por una nueva norma emocional, la solidaridad; y por la defensa de una concepción del obrero como ser humano digno y que los estos sintieron atacado dadas sus condiciones de existencia. Este ataque generó indignación y rabia, que fueron expresadas a través de diferentes prácticas. También se caracterizó por privilegiar la sociabilidad en las tabernas, que se convirtió en un refugio emocional socialista.
\end{abstract}

\section{Palabras clave}

Historia de las emociones - estudios del movimiento obrero - socialismo vizcaíno - régimen emocional socialista rojo - obreros mineros.

\begin{abstract}
The aim of this work is to study in a new way the making of class consciousness in the miner and industrial Biscay at the end of nineteenth century. We will focus on socialist movement, due to its predominance in this process, and we will use the theoretical and methodological tools provided by the history of emotions. Our proposal is to analyze class consciousness paying attention to the emotional expressions, practices and rituals, all of them constitutive elements of what we call Red socialist emotional regime, which underpins the socialist political program in this area. This regime was defined by the peaceable behavior during the demonstrations; by a new emotional norm, the solidarity; and by the defense of the idea of worker as a human being with dignity. The attack to that notion produced indignation and rage, which were expressed through some practices, as striking. This regime was also characterized by favoring the tavern as an emotional refugee.
\end{abstract}

\section{Keywords}

History of emotions - working class studies - socialism in Biscay - Red socialist emotional regime, miner workers. 


\section{Resumo}

Este artigo propõe um estudo renovada da formação da consciência de classe nos setores de mineração e industrial Vizcaya de finais do século XIX, com foco no movimento socialista, sua hegemonia neste processo, e usando Vandaimes teórico e metodológico da história as emoções Propomos analisar a consciência de classe através do estudo das expressões emocionais e práticas rituais que acreditamos que constituem o que chamamos de regime socialista vermelho emocional, formação emocional do programa político socialista nesta área. Este regime foi definido pelo pacifismo em atos de protesto; por uma nova norma emocional, solidariedade; e para a defesa de uma concepção do trabalhador como um ser humano digno e que se sentissem agredidos, dadas as suas condições de existência. Esse ataque gerou indignação e raiva, expressas através de diferentes práticas, dentre as quais destacam-se as greves. Caracterizou-se também por privilegiar a sociabilidade nas tabernas, que se tornou um refúgio emocional.

\section{Palavras-chave}

História das emoções - estudos do movimento operário - socialismo biscainho - regime emocional socialista vermelho - trabalhadores da mineração.

"No es la belleza de los discursos lo que nos cautiva. El socialismo bilbaíno no cuenta en sus filas ningún intelectual... ¿Para qué? En la brega, frente al capataz que les maltrata, frente al contratista que les obliga a surtirse en su tienda, frente al patrono que les merma el salario, no necesitan aquellos obreros cerebros superiores que edifiquen en párrafos soberbios la 'Ciudad del Buen Acuerdo', sino hombres decididos que endurezcan su valor y les arrastren a la lucha. Una vulgaridad cualquiera (...) sacude convulsivamente, bajo el cielo parduzco, entre las minas abandonadas y silenciosas, la roja multitud de los trabajadores (...), caiga sobre Bilbao la nube de gente roja, descargando torrencialmente las negruras condensadas."

Estas palabras, escritas por un joven Ramiro de Maeztu, nos muestran algunos de los elementos sobre los que pivotó el proceso de formación del movimiento obrero vizcaíno, entre los cuales no se citan las contradicciones económicas del capitalismo o la lucha por derechos políticos, sino que nos remite a la experiencia del maltrato, el valor, la lucha, o la acción. Precisamente la propuesta teórica de este trabajo defiende que la conciencia de clase no es un producto exclusivo de las condiciones económicas, de códigos culturales o de andamiajes discursivos, sino que una categoría hasta ahora marginada en estos estudios resulta central en su formación: la emoción.

A lo largo de este análisis del proceso de formación del movimiento obrero vasco, que va de la mano del Partido Socialista, veremos cómo la emoción es un elemento fundamental en ese proceso, y lo haremos analizando dos elementos clave: la huelga minera de 1890, acontecimiento fundacional del movimiento obrero vasco, y la sociabilidad en la taberna obrera, uno de los rasgos identificativos e identitarios de este primer socialismo.

\footnotetext{
* Fecha de recepción: 05/07/2019. Fecha de aceptación: 04/05/2020.

** Doctora en Ciencias Políticas, https://orcid.org/0000-0002-5564-7077.Departamento de Historia Contemporánea, Universidad del País Vasco/Euskal Herriko Unibertsitatea, Barrio Sarriena s/n, 48940 Leioa, Bizkaia, España, sara.hidalgo@ ehu.eus. Este trabajo forma parte de las investigaciones desarrolladas por el Grupo de Investigación del Sistema Universitario Vasco de Historia Social y Política del País Vasco Contemporáneo (IT-708-13), en el marco del proyecto "Nacionalización, Estado y violencias políticas. Experiencias, discursos y practicas (siglos XIX- XXI)" HAR2017-83955-P, del Ministerio de Economía y Competitividad.

${ }^{1}$ MAEZTU, 1899, 2.
} 


\section{La emoción: una categoría analítica para el estudio del movimiento obrero}

La historia de las emociones es una corriente que en los últimos años está teniendo un gran auge, renovando los estudios sobre el pasado, abriendo nuevos interrogantes sobre cómo nos acercamos a él y situando en el centro del análisis histórico a la emoción. Aunque su aplicación al estudio del movimiento obrero ha sido escasamente explorada, el estudio de cómo y por qué en determinados contextos se forma la conciencia de clase ha ido modulando desde los clásicos trabajos del materialismo histórico, que se dejaron de lado ante el empuje de la propuesta de E.P. Thompson, tanto con su categoría de experiencia ${ }^{2}$ como la de "moral economy". ${ }^{3}$ Arrancó así el giro cultural, al que le siguió el giro lingüístico ${ }^{4}$, hasta llegar al actual debate sobre el papel de las emociones en este proceso. $^{5}$

La emoción, el sentimiento o la pasión han tenido diferentes significados a lo largo de la historia. ${ }^{6}$ En la época inaugurada por la Revolución Francesa la emoción fue asimilada como opuesta a la razón, y asociada con lo femenino, el ámbito privado, lo volátil, lo irracional o lo inestable. Estos argumentos han sido rechazados en las últimas décadas, y a día de hoy numerosos estudios, provenientes de distintas disciplinas, demuestran que la emoción no solamente sustenta la racionalidad humana, ${ }^{7}$ sino que es una categoría historiable, culturalmente construida, lingüística o corporalmente expresada, y por tanto, susceptible de cambios.

La corriente historiográfica conocida como historia de las emociones, que recoge en gran parte lo expuesto en el párrafo anterior, afirma que éstas son una categoría útil, pertinente y renovadora para el estudio de la experiencia humana en el pasado. En este trabajo las emociones nos interesan no tanto como elementos que conforman la experiencia interna e individual del individuo, sino porque éstas son relacionales y nos muestran relaciones de poder, sociales, de género, de clase, y códigos culturales. Es decir, cómo se expresan las emociones, quién las expresa, dónde y ante quién son las preguntas que alumbran muchas respuestas para los trabajos históricos relativos al movimiento obrero. $^{8}$

La emoción como una categoría analítica para el análisis histórico fue propuesta por Lucien Febvre, en Sensibility and History en $1941 .{ }^{9}$ En los años cincuenta y sesenta este paradigma sufrió un cierto estancamiento debido al asentamiento de lo que se ha llamado el "racionalismo de posguerra", producido en gran medida por cómo se interpretaron los totalitarismos que desembocaron en la Segunda Guerra Mundial. ${ }^{10}$ No fue hasta 1970 cuando resurgió el interés por las diferentes dimensiones de la vida afectiva de las poblaciones en el pasado. En este ámbito destacaron los trabajos antropológicos que cuestionaban la universalidad de las emociones - poniendo por tanto el foco en su construcción cultural- y la noción hegemónica de que las emociones pertenecerían a lo

\footnotetext{
2 THOMPSON, 1964. Thompson ahonda en este concepto en THOMPSON, 1981

${ }^{3}$ THOMPSON, 1971.

${ }^{4}$ STEDMAN JONES, 1983; SCOTT, 1987.

${ }^{5}$ HAKE, 2017, HIDALGO, 2018.

${ }^{6}$ La definición de lo que hoy día entendemos por emoción ha cambiado a lo largo de la historia. En este trabajo usamos el concepto emoción para referirnos a todo ámbito opuesto a lo que se considera razón, ya que no hacemos una genealogía de la palabra. Sobre esto véase: FREVERT, 2014.

${ }^{7}$ Pionero en esta visión fue el neurólogo Antonio Damasio. DAMASIO, 1994.

${ }^{8}$ MATT y STEARNS, 2014.

${ }^{9}$ FEBVRE, 1973.

${ }^{10}$ Esta corriente entendía que los regímenes totalitarios de los años 1920-30 hasta 1945 habían tenido en la exaltación extrema de las emociones un elemento central. BIESS y GROSS, 2014.
} 
femenino - y por tanto la razón a lo masculino-. ${ }^{11}$

Paralelo a estos estudios, en otras disciplinas, la dicotomía entre razón y emoción fue encontrando cada vez más objeciones, al tiempo que se reubicó a esta última como elemento central en la conformación de la racionalidad humana y los procesos de toma de decisiones. Destacan aquí las investigaciones en psicología cognitiva ${ }^{12}$ y neurociencia ${ }^{13}$, ofreciendo esta última un corpus médico a los planteamientos filosóficos que entroncaban con Spinoza, la Ilustración escocesa o la fenomenología. ${ }^{14}$

La historiografía fue poco a poco haciéndose eco de estos estudios. Nos interesa especialmente el trabajo del historiador William M. Reddy quien, en 2001, publicó The Navigation of Feeling, una relectura de la Revolución Francesa bajo el prisma de la historia de las emociones, donde exponía un amplio y completo marco teórico que proporcionaba una serie de herramientas conceptuales muy útiles para el estudio de los procesos políticos en el pasado, como son el emotive y régimen emocional. En su análisis, Reddy distingue entre la emoción sentida y su expresión, ya que considera que la expresión emocional es un proceso complejo culturalmente mediado y que no consigue captar a través del lenguaje la totalidad del significado de la experiencia emocional. Así, la experiencia emocional se produciría en dos niveles: el nivel preconsciente, prelinguístico y precultural, que él llama emoción; y el nivel formado en la cultura y en el contexto, que denomina emotive. Éste es la traducción o "descripción" en palabras de una determinada acción que ocupa nuestra atención en un momento dado y de otras acciones que están en la retaguardia. Los emotives tienen tanto capacidad descriptiva de la emoción sentida, como performativa, ya que pueden transformar la emoción a la que se refieren, y por tanto son instrumentos que, con más o menos éxito, cambian, construyen, esconden o intensifican emociones. Este concepto lleva intrínseca una interesante implicación política, ya que en el esfuerzo por salvar esa división se situaría el ejercicio de poder, el cual englobaría tanto el control de un determinado régimen político, como el ámbito desde el que cambiar ese régimen. ${ }^{15}$ Es decir, en el espacio entre lo sentido y lo expresado se situaría la agencia humana y la capacidad de cambio histórico y político.

El segundo concepto reddydiano que nos interesa, y que de hecho nos sirve como encuadre teórico en este trabajo, es el de régimen emocional, definido como el conjunto de emociones normativas y rituales oficiales, prácticas y emotives que expresan e inculcan estas prácticas; siendo éste el necesario basamento de cualquier régimen político estable. Hay que tener en cuenta que para que un régimen emocional funcione no sólo es necesario que sea coherente con la configuración cultural de ese tiempo-espacio. La expresión emocional de un determinado régimen ha de evocar de manera exitosa en los y las participantes respuestas que ellos y ellas reconozcan y que garantizan estas expresiones. Este éxito es algo que ni la cultura ni el discurso pueden asegurar, y por ello tiene un gran significado político e histórico. En el caso de que un régimen emocional lleve a lo que él denomina sufrimiento emocional -es decir, a una disconformidad con los objetivos y los modos de relación con el mundo- se produce un proceso de autoexploración que lleva a la búsqueda de nuevas formas de expresión emocional. Esa búsqueda puede poner fin a un determinado régimen emocional $\mathrm{y}$, por tanto, al sistema político que sobre él se

\footnotetext{
${ }^{11}$ ROSALD, 1980; ABU-LUGHOD, 1986.

${ }^{12}$ LAZARUS, 1982.

${ }^{13}$ DAMASIO, 1994.

${ }^{14}$ Sobre la Ilustración escocesa véase: FRAZER, 2010. Sobre la filosofía y las emociones véase: GROSS, 2007.

${ }^{15}$ REDDY, 2001, 105.
} 
sustenta. ${ }^{16}$

Por otra parte, y en relación a la idea de la emoción como elemento explicativo clave de los procesos de cambio histórico o político, entendemos que ésta está intrínsecamente unida a los objetivos de los individuos. Así, las decisiones personales no son producto de una deliberación racional o de una medición cuantitativa de los intereses, sino también de las emociones que esa idea genera en el individuo. En este punto nos apoyamos en la propuesta del sociólogo Randal Collins quien afirma que la dinámica social es primeramente emocional, ya que el individuo decide a qué movimiento adherirse, no tanto por un cálculo racional del coste-beneficio, sino por lo que él llama el flujo/energía emocional (emotional flow) que esa dinámica genera. ${ }^{17}$

Las herramientas teóricas mostradas nos permiten proponer una relectura del proceso de formación de la conciencia de clase en la Vizcaya finisecular entendiendo ésta no como un producto exclusivo de la industrialización, de la pobreza o del lenguaje internacionalista, sino también como una propuesta sobre formas de gestión y expresión emocional, así como una forma de expresión de las emociones obreras de la experiencia social y política del momento. Es decir, proponemos entender la conciencia de clase y la formación del movimiento obrero en términos de regímenes emocionales.

\section{Experiencia y emoción obrera en la Vizcaya industrial y liberal de finales del XIX}

La clase obrera y el socialismo vizcaíno se desarrollaron en un contexto particular, el de la industrialización y modernización de esta región. En 1886 Bilbao y los 14 kilómetros de ría que la separan de la desembocadura, eran una mezcla de explotaciones mineras en los montes de Triano-Somorrostro e industrias siderometalúrgicas que pronto se convirtieron en el símbolo característico de la primera industrialización vizcaína. A nivel sociopolítico, aquí nació lo que más tarde se llamó el "pluralismo político vasco" conformado por el liberalismo dinástico, ${ }^{18}$ el socialismo ${ }^{19}$ y el nacionalismo vasco ${ }^{20}$-, la diversidad social y cultural, la modernidad y una sociedad de masas. ${ }^{21}$

Al mismo tiempo, a nivel social se produjeron cambios de calado. El fuerte flujo inmigratorio generó un brusco cambio demográfico que influyó negativamente en los modos de vida, cada vez más difíciles, de la población. ${ }^{22}$ La falta de vivienda fue una constante, así como el subsecuente hacinamiento, ausencia de higiene y falta de agua potable. ${ }^{23}$ Se generó así el caldo de cultivo idóneo para la propagación de enfermedades respiratorias y estomacales, que hicieron estragos entre la población ${ }^{24}$, unas enfermedades que no solamente tenían un origen médico, sino, sobre todo, social.

A nivel emocional, existía un debate sobre qué tipo de gestión emocional tenía la legitimidad política, de modo que la batalla política no solamente se tradujo en batalla programática, sino también emocional, es decir, qué emociones se relacionaron con qué

\footnotetext{
${ }^{16}$ REDDY, 2001, 118

${ }^{17}$ COLLINS, 2001, 41.

${ }^{18}$ ARANA, 1982, 13-32.

${ }^{19}$ FUSI, 1975 y HIDALGO, 2018.

${ }^{20}$ Aunque el nacionalismo vasco nace como reacción a este proceso modernizador, al constituirse en este momento y tener importancia política, se ha integrado en este grupo. CASTELLS, 1997.

${ }^{21}$ FUSI, 1990 y FUSI, 1990b, 264.

${ }^{22}$ Entre 1877 y 1887 el área metropolitana de Bilbao, pasó de los 62.417 a los 105.325 habitantes. El 84,7\% de este crecimiento correspondía a la inmigración y un 15,3\% al crecimiento natural (vegetativo). La mayor parte de este crecimiento (el $86,6 \%$ ) se concentró en los municipios mineros e industriales. GONZÁLEZ PORTILLA, 2001, 130 y 168.

${ }^{23}$ BEASCOECHEA, 2003; NOVO, 2002.

${ }^{24}$ GONZÁLEZ PORTILLA, op. cit. 306-312.
} 
colectivos. Las citadas tres culturas políticas del momento pugnaron también en este terreno, que se convirtió en un campo más de lucha política.

En este contexto dos colectivos sociales y políticos emergieron y trataron de definir la nueva realidad: la burguesía y el proletariado. La burguesía definía esta nueva problemática como "cuestión social", y defendía el "armonicismo social" y el paternalismo industrial como respuesta a los acuciantes desafíos que iban apareciendo. ${ }^{25}$ Por su parte, el grupo que más tarde dio cuerpo a la clase obrera, integraba la amalgama de trabajadores de las minas, fabriles y artesanos cada vez más depauperado, que experimentaban un contexto que no les favorecía y que poco a poco fueron unificando intereses hasta constituirse en sujeto político. ${ }^{26}$

Ahora bien, como se ha subrayado, entendemos que la formación de una conciencia política no es solamente producto de un contexto económico, cultural o político. Tampoco es consecuencia de la resistencia o resignificación discursiva a una interpelación negativa o estigmatizante. Defendemos, en cambio, que la clase como sujeto político se formó en torno a un régimen emocional que cuestionó el vigente, que no era otro que el régimen emocional burgués, basamento emocional del liberalismo. ${ }^{27}$

En efecto, durante el último tercio del XIX en Vizcaya la burguesía se asentó como un grupo hegemónico en lo social y político. Este sistema se sustentaba en lo que denominamos régimen emocional burgués, que, entre otros aspectos, se caracterizaba por expresar dos emociones, el miedo y el asco, altamente estigmatizadoras hacia el mundo obrero. Estas emociones, por supuesto, ni eran exclusivas de este colectivo ni se habían creado en este momento, pero nos interesan porque tomaron una forma política muy concreta y sirvieron a la burguesía para restar legitimidad al naciente movimiento obrero. La burguesía, al definir desde fuera a los obreros, lo hacía con un discurso basado tanto en el asco hacia el cuerpo y el modo de vida obreros como en el miedo a su ira y a su posible movilización de masa. En este sentido, Sabine Hake, en su análisis de la clase obrera alemana, detalla el modo en que los trabajos de teóricos como Friedrich Harkort o Gustave Le Bon, influyeron hondamente sobre una burguesía decimonónica que tenía todavía muy presentes tanto los sucesos revolucionarios de 1789 como los que jalonaron todo el siglo XIX. ${ }^{28}$

En el caso de la burguesía española en general y de la vizcaína en particular, el miedo se dirigía hacia la pobreza, la enfermedad del cuerpo obrero y hacia el creciente auge del internacionalismo, ${ }^{29}$ mientras que el asco se enfocaba hacia el modo de vida y estado del cuerpo obrero. Ambas emociones denotaban rechazo, y para entender la naturaleza constructivista del mismo, es necesario traer aquí el planteamiento de la socióloga Sarah Ahmed, según el cual tanto el miedo como el asco construyen el objeto que genera tal emoción, transformando al mismo tiempo al sujeto y al objeto. ${ }^{30}$ En este proceso, el obrero fue creado y percibido por la burguesía como animal, irracional, por tanto, un sujeto peligroso y rechazable, lo cual, traducido políticamente, restaba legitimidad a su proyecto político al tiempo que lo situaba en un grado inferior al proyecto liberal. A modo

\footnotetext{
${ }^{25}$ CAPELLÁN DE MIGUEL, 2004, y SIERRA, 1990.

${ }^{26}$ RUZAFA, 1998.

${ }^{27}$ Este trabajo no tiene como objetivo analizar el régimen emocional burgués, aquel correspondiente al liberalismo burgués y cuya preponderancia en Vizcaya se produce en el último tercio del XIX. Someramente expondremos en cambio las dos emociones que este régimen mantiene hacia el colectivo obrero.

${ }^{28}$ HAKE, 2017, 33-34.

${ }^{29}$ Sobre el miedo de la burguesía al creciente poder a los trabajadores como un colectivo véase PÉREZ LEDESMA, 1993, 28.

${ }^{30}$ AHMED, 2004. 62-64 y 88-97.
} 
sintético, hay que resaltar dos acontecimientos que expresaron de manera clara esta visión.

El primero fue la puesta en marcha de la Comisión de Reformas Sociales en 1883, en que por primera vez el gobierno liberal envió una encuesta a todo el país para conocer la situación de las clases populares. ${ }^{31}$ Esta comisión emanó de la burguesía más progresista y la encuesta resultó altamente interpeladora, seguramente porque como nos recuerda Pierre Bourdieu, el poder de las palabras reside en el capital simbólico acumulado por quien las nombra, ${ }^{32}$ y la burguesía del momento, sin duda, acumulaba tal capital. En la Comisión, además, por primera vez, los socialistas dialogaron directamente con la burguesía en la tribuna pública, y en ella actuó como ponente el líder socialista de Vizcaya, Facundo Perezagua. Por una parte, este ente fue reflejo del miedo que la burguesía sentía hacia el cada vez más pujante internacionalismo - "siente el capital inquietudes justificadas por hondas y continuas perturbaciones"-,${ }^{33}$ y por ello quedó adscrita al Ministerio de Gobernación. Por otra parte, evidenció el profundo desprecio de la burguesía hacia las clases populares y sus modos de vida. Un ejemplo sintomático de esto último fue el informe de la Institución Libre de Enseñanza, que aglutinaba a la burguesía más progresista del país, y que se refería al obrero de la siguiente manera:

"la ignorancia casi absoluta es su patrimonio, y embotado el sentimiento y desarreglada su voluntad, ofrecen un conjunto de carácter semisalvaje y primitivo (...) su sistema nervioso dispuesto todo exceso o un sentimiento de semiidiotismo que las incapacite para todas las relaciones individuales y sociales." 34

El segundo acontecimiento que nos muestra cómo se expresaron las emociones de rechazo de la burguesía hacia el mundo obrero fueron las medidas tomadas durante la epidemia de cólera de 1885, que sacudió a la provincia de Vizcaya, y que nos deja pruebas de una primera resistencia obrera a ser definidos como enfermos, sucios, inmorales y animalizados. El cólera de 1885 llegó a Vizcaya más tarde que al resto de España, donde la epidemia ya había empezado a remitir y cuando se creía que la provincia se había librado del mal. ${ }^{35}$ No obstante, cuando empezaron los primeros casos, las zonas obreras vieron cómo la enfermedad se expandía con rapidez por sus municipios. Sin duda el hacinamiento y la falta de medidas higiénicas favorecieron la propagación, pero las autoridades del momento entendieron que la inmoralidad y animalidad que presuponían a los obreros eran las responsables, reforzando así tanto el miedo como la implícita emoción del asco. Ilustrativas de esta idea son estas palabras, "la causa de la enfermedad son las repugnantes manifestaciones de corrupción y miseria moral". ${ }^{36}$ Por ello durante esta epidemia las autoridades liberales del momento crearon un cordón sanitario que segregaba a las poblaciones mineras e industriales, ocupando "un perímetro de 45 kilómetros desde el extremo del río Cadagua a Somorrostro". "Un cordón que no

\footnotetext{
${ }^{31}$ CASTILLO, 1985.

${ }^{32}$ BOURDIEU, 2008, 89.

33 "Real Decreto creación Comisión de Reformas Sociales", Gaceta de Madrid, tomo IV, no 344, 10 de diciembre 1883, 761-762

${ }^{34}$ CASTILLO, op.cit. Tomo II, 274 y 289

35 El colectivo médico revestía sus análisis del cólera no solamente con planteamientos médicos, sino también moralistas. Así, además de "por estar bien ventilado" se planteaba que Vizcaya estaba protegido del cólera porque "este pueblo es aseado, trabajador y morigerado". GIL Y FRESNO, 1884, 4.

${ }^{36}$ El Noticiero Bilbaíno, 20 de agosto de 1885, 1.

${ }^{37}$ El Noticiero Bilbaíno, 31 de octubre de1885, 1. Asimismo, en la prensa local fueron frecuentes titulares como "Terror por el cólera" o explicaciones como que la enfermedad la había traído a la ciudad "una mendiga" venida de fuera. El Noticiero Bilbaíno, 17 de agosto de 1885, 1 al 27 de agosto de 1885, 1.
} 
solamente tenía un origen médico sino también social, dado que aislaba a las poblaciones obreras y mineras. Asimismo, se afirmaba no solamente que la enfermedad tenía su origen en las zonas obreras, sino que se explicaba su aparición por las "detestables" condiciones higiénicas en que vivían en la zona minera, adonde llegaban a diario personas de otras regiones "sin precauciones". ${ }^{38}$ Estas medidas supusieron una interpelación muy fuerte y directa hacia grupo poblacional que entendió que ese aislamiento llevaba intrínseco la estigmatización y el cuestionamiento de su humanidad. Así, las poblaciones obreras no sólo no aceptaron el citado cordón sanitario, sino que muchos lo sobrepasaron para escapar, generando el pánico entre la población de fuera del mismo y siendo descritos con epítetos como "bandadas de obreros", ${ }^{39}$-lo cual reforzaba el discurso que equiparaba al obrero con lo irracional, lo peligroso o lo animal-.

La respuesta del mundo obrero no se hizo esperar, y, en el caso vizcaíno, estuvo articulada en torno al socialismo y a la noción de clase, así como al régimen emocional que lo sustentaba. Hay que recordar que el partido socialista se había creado en 1879 en Madrid, y en 1886 en Bilbao, aunque hubo de esperar hasta 1890 para convertirse en un movimiento de masas. Así pues, defendemos que el primer socialismo vizcaíno no solamente fue una propuesta programática, sino también emocional, es decir, fue una forma de expresión de las emociones de indignación y rabia que conformaban la experiencia obrera descrita, y estuvo sustentado por lo que hemos llamado régimen emocional socialista rojo, ${ }^{40}$ el conjunto expresiones emocionales, de normatividades emocionales, rituales oficiales y prácticas que el colectivo obrero mantuvo. Este régimen se definió por el pacifismo en los actos reivindicativos; por una norma emocional que cimentaba el sentimiento de comunidad, la solidaridad; y por la defensa de un código de dignidad obrero, que englobaba una concepción del obrero como ser humano digno y que los obreros sintieron atacado dadas sus condiciones de vida. Las prácticas más habituales de este régimen fueron las huelgas (forma de expresión colectiva de la indignación y la rabia), las llevadas a cabo en el espacio de la taberna -una de las particularidades del socialismo vizcaíno- así como los rituales del $1^{\circ}$ de mayo. Este régimen también se definió por las emociones estigmatizantes proyectadas sobre el trabajo femenino, aunque un pormenorizado análisis de las diferencias de género sobre las que se asienta la conciencia de clase no se hará en este trabajo. ${ }^{41}$ Así, consideramos que la clase y el movimiento obrero vizcaíno nacieron con este régimen emocional durante los acontecimientos de mayo de 1890.

\section{De la emoción obrera a la política socialista: mayo de 1890}

En mayo de 1890 se produjeron los acontecimientos fundacionales de la clase obrera vizcaína y de la hegemonía del partido socialista: el $1^{\circ}$ de mayo y la huelga minera. Estos hechos han sido profusamente estudiados por la historiografía, ${ }^{42}$ y aquí presentamos una relectura de los mismos entendiéndolos como un modo de expresión de las emociones que la experiencia del nuevo contexto de industrialización y modernización generó en una parte importante del mundo obrero.

\footnotetext{
${ }^{38}$ El Noticiero Bilbaíno, 27 de octubre de 1885, 1.

${ }^{39}$ Ibidem, 3 de noviembre de1885, 1.

${ }^{40}$ Se ha decidido denominar así este régimen emocional respetando el modo en que en la época definían a los mineros debido al color que adquirían durante su trabajo en las minas: rojos. Valga como ejemplo las palabras del escritor socialista Julián Zugazagoitia al referirse a éstos: "Roja la color y rojas las ideas y el alma. Tal son los mineros vizcaínos”, ZUGAZAGOITIA, 1922, 2.

${ }^{41}$ Sobre la definición que el régimen emocional socialista rojo y el primer socialismo vizcaíno hicieron del trabajo femenino véase: HIDALGO, 2018, op. cit. 120-133; 184-207 y 244-256.

${ }^{42}$ FUSI, 1975, op. cit. 81-94, MIRALLES, 1990, HIDALGO, 2015.
} 
Se han presentado algunos elementos de la experiencia obrera en aquel momento y cuáles fueron los elementos emocionales sobre los que pivotó el discurso de la burguesía. Asimismo, se ha señalado que la idea de humanidad y dignidad fueron centrales tanto para entender las emociones que generó el contexto en los obreros como para comprender su respuesta.

Antes de continuar con la huelga es necesario subrayar que una parte importante del mundo obrero tenía una noción dignidad cuyo significado habían trasladado al mundo industrial desde el campo y el mundo artesanal, de donde provenían una gran parte de los obreros. ${ }^{43}$ Esta percepción consideramos que se engloba en lo que hemos denominado un código de dignidad. ${ }^{44}$ Nuestro análisis nos ha llevado a proponer la idea de que los obreros experimentaron una honda contradicción entre este código y su experiencia del día a día, especialmente en las minas, donde los obreros, para conseguir ser contratados, generalmente tenían que hospedarse y proveerse de víveres en los barracones y cantinas previamente acordados con el capataz, que se llevaba una comisión por el negocio, y cuyo pago se descontaba directamente del sueldo, encadenando muchas veces a los obreros a una deuda. Éstos sintieron que esta situación atacaba frontalmente su código de dignidad, lo cual generó indignación, rabia, y un fuerte sufrimiento emocional, término analítico reddydiano muy útil en este trabajo y que se refiere a un agudo conflicto de intereses que puede propulsar el cambio. ${ }^{45} \mathrm{El}$ socialismo vizcaíno por tanto no introdujo esta noción en los obreros, sino que operó en un campo fértil al hacer suya la defensa de este código, resignificándolo en ciertos aspectos, y canalizando estas emociones a través de su ideario, todo lo cual, al ser secundado por un número significativo de obreros, convirtió al socialismo y al colectivo minero en hegemónicos del primer movimiento obrero vasco.

Durante la manifestación del día del trabajo en Bilbao - que se celebró el 4 de mayo, domingo- ${ }^{46}$ una de las principales reivindicaciones fue la de la condición de humanidad: "queremos ser hombres y queremos ser libres", ${ }^{47}$ se gritaba durante los mítines. Asimismo, se afirmó que no era tanto una reivindicación salarial lo que les irritaba sino las condiciones de vida:

“contra lo que sí están irritados es contra la existencia de esos cuarteles donde se les obliga a proveerse en condiciones muy desfavorables, tanto por la calidad de los artículos como por el precio de los mismos. También se lamentan amargamente de que se vean precisados a dormir en barracas de malísimas condiciones, donde muchas veces yacen amontonados numerosos obreros con grave perjuicio para su salud y su vida". ${ }^{48}$

A los gritos espontáneos de “¡Abajo los cuarteles!” durante las marchas por Bilbao se le sumó la alocución de Perezagua ante 4000 personas, hombres y mujeres trabajadoras, donde censuró "los cuarteles y las barracas donde se explota a los mineros

\footnotetext{
${ }^{43}$ Ilustrativo de esta idea es que en 1876 la mecanización de las artes y oficios supuso un palpable deterioro de sus condiciones de trabajo, lo cual les llevó a protagonizar sonadas quejas y a reivindicar su dignidad, como la de 22 oficiales papeleros de La Peña, "todos ellos padres de familia" que en 1888 llevaron a cabo una protesta contra el nuevo director "una persona que carece todavía de los conocimientos necesarios para ello", "porque no nos parecía digno el estar bajo la dirección de quien sabe menos que nosotros" $\mathrm{El}$ Noticiero Bilbaíno, 14 de octubre 1888. Cita en RUZAFA, 1998, op. cit....p. 57

${ }^{44}$ Uso el concepto "código de dignidad" inspirado por Robert Nye y su concepto de "código de honor" NYE, 1993, 7-13. Asimismo, para la cuestión de defensa de un determinado código ha sido de gran interés para este trabajo el concepto "moral economy" THOMPSON, 1971

${ }^{45}$ W.M. REDDY, 2001, op .cit., 129.

${ }^{46}$ RALLE, 1991, 74.

47 “La manifestación de hoy”, El Noticiero Bilbaíno, 4 de mayo de 1890, 1.

${ }^{48}$ Ibidem.
} 
haciendo con ellos heregías (sic) y tratándoles como bestias (Aprobación unánime)", ${ }^{49}$ lo cual ponía de relieve la indignación que generaba en los obreros ser asimilados a las alimañas.

Tras la fiesta del trabajo reinó una aparente normalidad en Bilbao y sus zonas industriales,${ }^{50}$ pero a tenor de lo que más tarde ocurrió, queda patente que algo cambió en la conciencia obrera. Y es que, a los pocos días, el 13 de mayo, un hecho que previamente habría sido considerado intrascendente, como fue el despido de cinco trabajadores por su participación en la fiesta del trabajo, fue entendido como inadmisible por los obreros e hizo estallar la huelga. Hemos conceptuado la huelga como una de las prácticas centrales del régimen emocional socialista rojo, al ser un elemento fundamental en la creación de la conciencia de clase en Vizcaya, ya que durante la misma se produjo comunicación e intercambio emocional, conditio sine qua non en el proceso de creación de una comunidad. Además, los trabajadores compartieron un estilo emocional, representaron un mismo régimen emocional y persiguieron unos objetivos comunes. El paro comenzó en la empresa La Orconera y a medida que pasaban las horas los adheridos se contaban por miles, así como los desafíos y desplantes que hacían a las fuerzas del orden y que dan cuenta de la sensación de fuerza colectiva que sentían. ${ }^{51}$ Sus reivindicaciones eran poner fin al truck system, menos horas de trabajo, supresión del sistema de tarea y readmisión de los despedidos. ${ }^{52}$ El partido socialista se erigió rápidamente en representante de los obreros, cosa que éstos aceptaron en una clara muestra de confianza hacia sus líderes.

Por otra parte, se necesitaba de una poderosa normatividad emocional que cimentara el sentimiento colectivo y esa fue precisamente la solidaridad, una emoción que no es exclusiva de la clase obrera pero que para la izquierda ha tenido un fuerte significado simbólico desde la Revolución Francesa ${ }^{53}$ y que el movimiento internacionalista usó de una manera muy efectiva como elemento conformador de su cultura política. ${ }^{54}$ La socióloga Deborah Gould afirma que la solidaridad es tanto un estado afectivo como un conjunto de prácticas, y la define como afinidades y reciprocidades a través de las diferencias. ${ }^{55}$ Durante la huelga de 1890 , la solidaridad se fue reforzando al compás de los acontecimientos, y provocó que trabajadores que no se conocían crearan lazos recíprocos de confianza, compartieran las mismas expresiones emocionales, percibieran la unicidad de intereses, y secundaran como una única colectividad a la huelga. De hecho, las fuentes muestran que la solidaridad operó entre los obreros vizcaínos, se asimiló con la lucha de clases y cimentó al colectivo:

"en primero lugar, ha afirmado gallardamente la solidaridad entre todos los explotados del mundo. Nadie que se tenga por serio podrá negar hoy que la inmensa masa obrera, sin distinción de nacionalidad ni de raza, tiene un programa común y se mueve impulsada por los mismos sentimientos". 56

Sin duda, la solidaridad fue crucial para consolidar el sentimiento de clase. Un ejemplo sintomático nos lo da el momento y las razones por las que los trabajadores

\footnotetext{
49 “La manifestación obrera de ayer”, El Noticiero Bilbaíno, 6 de mayo de 1890, 1.

${ }^{50}$ El Noticiero Bilbaíno, 10 de mayo de1890, 1.

${ }^{51}$ Algunos ejemplos de la actitud desafiante de los obreros en El Noticiero Bilbaíno, 17 de mayo de 1890, 1, El Guipuzcoano. Diario liberal reformista, 18 de mayo de1890, 1-8 y La Libertad (San Sebastián) 18 de mayo de1890, 1.

52 El Noticiero Bilbaíno, 17 de mayo de 1890, 1.

${ }^{53}$ DOMENECH, 2004.

${ }^{54}$ Sobre este concepto véase la interesante aportación de BARKER, 2001.

${ }^{55}$ GOULD, 2009, 328.

56 "Resultados de la manifestación internacional”, El Socialista, 16 de mayo de 1890, 1.
} 
metalúrgicos secundaron la huelga y apoyaron a los mineros: "nosotros estamos muy satisfechos (con los salarios). Trabajamos menos tiempo que los demás obreros, tenemos libres las tardes de los sábados y nuestros jornales son crecidos, pero tenemos espíritu de compañerismo y defendemos los derechos de nuestros hermanos de las minas". ${ }^{57}$ Hay que tener en cuenta además que, para garantizar que las emociones cohesionadoras operen, es necesario de un reverso, es decir, de emociones negativas que dejen fuera del colectivo a aquellos que no comparten o no se rigen por las normatividades emocionales consensuadas por una comunidad. En el caso que analizamos la solidaridad se garantizó con otra emoción, la vergüenza. Así, cuando los obreros llamaron al paro a los metalúrgicos lo hicieron al grito de "es de traidores abandonarnos (tras lo cual) los obreros todos se marcharon abandonando los talleres". 58 No olvidemos la negativa carga semántica y emocional del concepto traidor, aquel que rompe el grupo y pone en peligro los intereses comunes, epítome de la maldad, lo contaminado y lo contaminante. ${ }^{59}$

Además de las prácticas, el régimen emocional socialista rojo exhibió durante estos hechos un estilo/tono emocional ${ }^{60}$ pacífico que mantendría a lo largo de los años. Es cierto que esta afirmación puede contradecir la visión que se tenía de los mineros, de carácter hosco, oscuro y un tanto agresivo - carácter además bien representado por su líder, Facundo Perezagua-. ${ }^{61}$ Pero no es menos cierto que en este periodo, aunque se llevaron a cabo acciones con cierta dosis de violencia y existieron sabotajes y coacciones durante las huelgas, los obreros no protagonizaron acciones terroristas ni asesinatos, ${ }^{62}$ todo lo cual nos lleva a defender la denominación de pacifismo para referirnos al estilo emocional del primer socialismo vizcaíno. Esta característica ya se puso de relieve desde el principio de la huelga, cuando se afirmó que "mientras nosotros no ataquemos las instituciones vigentes estamos dentro de la ley. Iremos pacíficamente". ${ }^{63}$ Sin duda el socialismo era muy consciente de que en este campo se jugaba su legitimidad política y que protagonizar acciones violentas habría ahondado en la percepción de barbarie, irracionalidad y peligrosidad que la burguesía tenía sobre ellos. No extraña por tanto que para contrarrestar esto afirmaran que "los parásitos de la sociedad (la burguesía) tiemblan de espanto ante las pacíficas manifestaciones de los trabajadores del mundo civilizado". ${ }^{64}$ Retóricamente, el socialismo trataba de invertir el eje civilización-barbarie a favor de los obreros, cambiando por tanto la jerarquía de la legitimidad política.

La huelga finalizó el 20 de mayo de 1890 con el famoso Pacto Loma, previa intervención del ejército. El general Loma, que da nombre al pacto, cuyo regimiento había sido llamado para garantizar el orden en la zona minera, forzó a la patronal a aceptar las reivindicaciones obreras al tiempo que reconoció a los socialistas como interlocutores en el conflicto. Se ponía fin así a la huelga, pero también, al menos sobre el papel, al truck system. Al mismo tiempo, se iniciaba la hegemonía del socialismo en el movimiento obrero vizcaíno, consagrando al minero como icono de la clase y la acción de la huelga como una de las tácticas principales para la consecución de sus objetivos.

\footnotetext{
${ }^{57}$ La Libertad (San Sebastián), 18 de mayo de 1890, 1.

${ }^{58}$ Ibidem, 17 de mayo de1890, 2.

${ }^{59}$ Este concepto adquirirá cada vez más fuerza dentro del socialismo en las primeras décadas del siglo XX, siendo un concepto central para evitar que obreros rompieran la huelga en 1904 y 1910, asimilado también al de "esquirol". Véase HIDALGO, 2018, op. cit. 288-289.

${ }^{60}$ Aquí hacemos la traducción del inglés de "emotional tone". No estamos introduciendo el concepto, desarrollado por W. Reddy de "emotional styles". REDDY, 2008.

${ }^{61}$ HIDALGO, 2016.

62 Juan Pablo Fusi señala a este respecto que los socialistas vizcaínos recondujeron la violencia obrera hacia la negociación, es decir, actuaron como mediadores. FUSI, 1990, 67.

${ }^{63}$ El Noticiero Bilbaíno, 15 de mayo de 1890, 2.

64 “Manifestación internacional obrera. Bilbao", El Socialista, 23 de mayo de 1890, 2.
} 


\section{La taberna. Un refugio emocional para la clase obrera vizcaína}

En las zonas industrializadas la taberna jugó un papel fundamental como centro de sociabilidad obrera. ${ }^{65}$ "Los centros donde una gran parte de la sociedad pasa mucho de su vida" ${ }^{66}$ como apareció definida en la época, funcionó como espacio catalizador de nuevas experiencias, máxime en un momento en el que la gestión del tiempo libre estaba cambiando, consecuencia de la nueva disciplina horaria, y la actividad laboral resultaba más reificante que durante la época preindustrial. ${ }^{67}$ La taberna, además, se convirtió en un lugar donde las relaciones interpersonales se redefinieron, producto en parte de la disolución de los lazos familiares propia de la vida urbana que creaba otro tipo de hermanamientos, como podía ser el político. Además, la taberna fue un espacio con una fuerte carga emotiva, ya que se convirtió en un lugar donde crear confianzas personales, compartir experiencias personales (y las emociones que esas experiencias generaban), y estrechar lazos afectivos. Por último, no podemos olvidar que la taberna era un espacio con evidentes connotaciones de género, ya que se convirtió en el espacio masculino por excelencia, "el punto de reunión de los hombres", ${ }^{68}$ y las mujeres que a ella acudían por distintas razones, eran negativamente connotadas.

Por otra parte, y referido a la pugna política entablada entre el mundo obrero y la burguesía, la taberna se convirtió en un auténtico campo de batalla, ya que ésta entendió que las prácticas de este espacio reafirmaban el carácter irracional, temperamental, y, por ende, peligroso y violento del obrero.

"En el local de la taberna hay algo que ofende y predispone al delito (...). A medida que las libaciones se suceden, los instintos se exaltan: se bebe veneno y se respira veneno. (...) el instinto matón del pueblo domina a todo sentimiento; cada mirada es un reto, la palabra un ataque, toda contestación toma forma de navaja (...)" ${ }^{99}$,

afirmaba un fiscal refiriéndose a Asturias. Además, en sintonía con el pensamiento liberal, la taberna se convirtió en el origen de la miseria del obrero, a quien responsabilizaban de acudir a este espacio donde maltrataban su cuerpo y su mente. Así lo afirmaba el médico bilbaíno José Gil y Fresno:

"no olvidéis que la borrachera mata la fe conyugal, destruye la decencia y el recato; que un borracho no respeta la autoridad, ni lo sagrado ni lo profano; que con facilidad promueve pendencias, algunas sangrientas, y concluye, como todos los días estamos viendo, por ser el juguete de los niños y el desprecio de los mayores terminando su mísera existencia delirante y tembloroso en un hospital." 70

Dada su importancia para la cultura popular y los ataques que la burguesía hacía a la taberna, para el primer socialismo vizcaíno ésta fue un lugar contradictorio, en torno al cual mantuvo un frágil equilibrio. Es cierto que en sus principios programáticos, éste defendía que la ingesta de alcohol era un elemento más de la explotación del obrero, ya que alienaba y embotaba sus sentidos, convirtiéndose en una víctima de un sistema capitalista que tenía en la administración del alcohol una forma más de opresión. ${ }^{71}$ Pero,

\footnotetext{
${ }^{65}$ SERRANO, 1989, 22.; RALLE, 1989; SIERRA, 1994, 79.

${ }^{66}$ El Noticiero Bilbaíno, 6 de enero de 1890, 1.

${ }^{67}$ BURKE, 1995.

${ }^{68}$ VERGARA GACÍA, 1904, 145.

${ }^{69}$ GIMENO y AZCÁRATE, 1900, 52-53.

${ }^{70}$ GIL Y FRESNO, 1871, 87-88.

${ }^{71}$ CAMPOS, 1998.
} 
a pesar de ello, el socialismo vizcaíno, consciente de que la taberna era un lugar central para el mundo obrero al cual querían atraer a sus filas, hizo de este espacio un refugio emocional -término definido por William Reddy como el lugar donde se produce un relajamiento de las normas emocionales vigentes, donde se puede cuestionar el régimen emocional vigente (y su sistema político) y dar forma a uno nuevo- ${ }^{72}$ desde el que se cuestionó el régimen emocional burgués. Desde ese refugio emocional la decantación de la experiencia común compartida fue cristalizando en un movimiento político, reforzado además por la camaradería y confianza que generaban las asiduas visitas a este lugar. En Bilbao, las primeras casas del pueblo (sedes de la sociabilidad socialista) no aparecieron hasta la primera década del XX, y por tanto, la taberna regentada por Facundo Perezagua en el barrio obrero de las Cortes era, de facto, el centro obrero, tal y como recordaba, con cierto horror, el socialista madrileño Andrés Saborit: "la taberna de Perezagua (...) era un círculo socialista". ${ }^{73}$ No hay duda de que en las tabernas se hablaba de política y se discutían estrategias. Valgan como ejemplo el hecho de que la primera reunión que Perezagua mantuvo con unos mineros hacia 1887 se produjera en una de estas, ${ }^{74}$ o que fuera en este espacio, en los muelles de Bilbao, donde se decidiera secundar la huelga de $1890 .{ }^{75}$ No es casualidad que el privilegiar la taberna como un espacio político fuera uno de los rasgos distintivos del primer socialismo vizcaíno y del régimen emocional socialista rojo.

\section{Conclusiones}

A lo largo del trabajo se ha mostrado que las herramientas teóricas de la historia de las emociones resultan útiles y pertinentes para el estudio del movimiento obrero. El análisis del caso vizcaíno a finales del XIX demuestra que la emoción también fue fundamento de la conciencia de clase y del movimiento obrero. Una parte significativa del colectivo obrero no se adhirió al socialismo exclusivamente por reivindicaciones laborales o políticas, sino por las emociones que en ellos generó la experiencia de su realidad, las cuales fueron canalizadas y politizadas por el movimiento socialista, creando lo que hemos llamado régimen emocional socialista rojo, cuyas características han quedado expuestas.

El análisis de los procesos de formación del movimiento obrero al que nos llevan las herramientas proporcionadas por el giro emocional nos obliga también a una reflexión sobre cómo definimos y entendemos la propia clase, conclusión final de este trabajo. En sintonía con lo expuesto a lo largo del trabajo, rechazamos la idea de que la clase es el producto de los dictados del capitalismo, de la experiencia de la industrialización o la construcción identitaria que resulta del despliegue de unas determinadas categorías culturales o discursivas. Aunque somos deudores de las aportaciones del concepto de experiencia thompsoniano y las del giro lingüístico, creemos que una mejor comprensión de cómo y por qué surge la conciencia de clase debería considerar la complejidad de la experiencia humana. Proponemos así entender la conciencia de clase como la expresión

\footnotetext{
72 REDDY, 2001, op. cit. 128.

73 "Semblanza de Indalecio Prieto" El Socialista, 30 de abril 1953, 3.

${ }^{74}$ Las palabras de Perezagua al rememorar la primera vez que acudió a las minas, hacia 1887, a hacer propaganda, pensando que iba a encontrarse con una nutrida concurrencia y los únicos que acudieron fueron tres mineros y como "no era cosa de que ocupáramos la tribuna para predicar ante auditorio tan numeroso, y decidimos platicar de sobremesa, ¡una comida de propaganda!". En "Perezagua se decide por el sindicalismo" El Liberal, 3 de diciembre de 1914, 1.

${ }^{75}$ Esta era una práctica que se producía, a tenor de lo que se desprende de los expedientes judiciales, como este de 1890 que relata en una cantina situada en los muelles de Bilbao, "dentro y en la calle, había un gran grupo de personas (...) que decían hoy no se trabaja” AHFB. Fondo Judicial. JCR 623/038.
} 
de la respuesta emocional a la experiencia que una parte del colectivo obrero tuvo de los cambios sociales, económicos y políticos que se produjeron en el siglo XIX en zonas como la cuenca vizcaína del Nervión. Consideramos por tanto que la conciencia de clase en la cuenca del Nervión se formó en torno a un determinado régimen emocional, que hemos denominado socialista rojo. Fue entonces cuando las emociones obreras, se convirtieron en política socialista.

\section{REFERENCIAS BIBLIOGRÁFICAS}

Archivos

Archivo Histórico Foral de Bizkaia.

Fuentes primarias

El Guipuzcoano. Diario liberal reformista, 1890.

El Liberal, 1914.

El Noticiero Bilbaíno, 1885, 1890.

El Socialista, 1890.

Gaceta de Madrid, 1883.

La Libertad (San Sebastián), 1890.

MAEZTU, Ramiro, "Las minas de Bilbao", Vida Nueva, 30 de abril de 1899, pp. 1-4.

ZUGAZAGOITIA, Julián, “Triconomía obrera. Fábricas, minas y talleres”, El Liberal

(Bilbao), 20 de agosto de 1922, p. 2.

Bibliografía

ABU-LUGHOD, Lila, Veiled sentiments. Honor and poetry in a Bedouin society, California, University of California Press, 1986.

AHMED, Sara, The cultural politics of emotion, Edinburgh, Edinburg University Press, 2004.

ARANA, Ignacio, El monarquismo en Vizcaya durante la crisis del reinado de Alfonso XIII (1917-1831), Universidad de Navarra, Pamplona, 1982.

BARKER, Colin, "The Making of Solidarity at the Lenin Shipyard in Gdansk" en GOODWIN, James, JASPER, James y POLLETTA, Francesca, Passionate Politics. Emotions and social movements, Chicago, Chicago University Press, 2001, pp. 175195.

BEASCOECHEA, José María, "Jerarquización social del espacio urbano en el Bilbao de la industrialización" Scripta Nova. Revista electrónica de geografía y ciencias sociales, 146, (2003), pp.1-16.

BIESS, Frank y GROSS, Daniel (eds.), Science and Emotions after 1945, Chicago, University of Chicago Press, 2014.

BOURDIEU, Pierre, ¿Qué significa hablar? Economía de los intercambios lingüísticos, Madrid, Akal, 2008.

BURKE, Peter, "The invention of Leisure in Early Modern Europe", Past and Present 146, (1995), pp. 136-150. 
CAMPOS MARÍN, Ricardo, "El obrero abstemio. Salud moral y política en el discurso antialcohólico del socialismo español a principio de siglo", Historia Social, 32, (1998), pp. 27-43.

CAPELLÁN DE MIGUEL, Gonzalo, "Cambio conceptual y cambio histórico. Del pauperismo a la cuestión social” Historia Contemporánea, 29, (2004), pp. 539-590.

CASTELLS, Luis, "El nacionalismo vasco (1890-1923): ¿una ideología modernizadora?", Ayer, 28, (1997), pp. 127-162.

CASTILLO, Santiago, Reformas Sociales, Madrid, Ministerio de Trabajo y Seguridad Social, 1985.

COLLINS, Randal, "Social movements and the focus of Emotions Attention" en GOODWIN, James, JASPER, James y POLLETTA, Francesca, Passionate Politics. Emotions and social movements, Chicago, Chicago University Press, 2001, pp. 27-45.

DAMASIO, Antonio, Descartes' Error. Emotion, reason and human brain, New York, Avon Books, 1994.

DOMENECH, Antoni, El eclipse de la fraternidad. Una revisión republicana de la tradición socialista, Barcelona, Crítica, 2004.

FEBVRE, Lucien, "Sensibility and History: How to Reconstitute the Emotional Life of the Past", en BURKE, Peter (ed.), A New Kind of History, London, Harper Row, 1973, pp. 12-26.

FRAZER, Michael, The Enlightenment of Sympathy. Justice and the Moral Sentiments in the Eighteenth Century and Today, New York, Oxford Universtity Press, 2010.

FREVERT, Ute, Emotional lexicons: continuity and change in the vocabulary of feeling 1700-2000, New York, Oxford University Press, 2014.

FUSI, Juan Pablo, Política obrera en el País Vasco, Madrid, Taurus, 1975.

----- El País Vasco. Pluralismo y nacionalidad, Madrid, Alianza, 1990

----- "La edad de las masas", Historia contemporánea, 4, (1990), pp. 261-272.

GIL Y FRESNO, José, Higiene física y moral del bilbaíno. Bilbao, Imprenta de Juan E. Delmas, 1871.

GIL Y FRESNO, José "Cartas de un médico viejo. Acerca del cólera”, El Noticiero Bilbaíno, 28 de julio de 1884, p. 4.

GIMENO Y AZCÁRATE, Manuel, La criminalidad en Asturias. Estadística (18831897), Oviedo, Escuela Tipográfica del Hospicio, 1900.

GONZÁLEZ PORTILLA, Manuel (ed.), Los orígenes de una metrópoli industrial: la ría de Bilbao vol. I, Bilbao, Fundación BBVA, 2001.

GOULD, Deborah, Moving politics. Emotion and Acts Up's fight against AIDS, Chicago, Chicago University Press, 2009.

GROSS, Daniel, The secret history of emotion. From Aristotles Rhetoric to Modern Brain Science, Chicago, Chicago University Press, 2007.

HAKE, Sabine, The proletarian dream. Socialism, culture and emotion in Germany, 1963-1933, Berlin, DeGruyter, 2017.

HIDALGO GARCÍA DE ORELLÁN, Sara, "Emociones socialistas en la huelga minera de 1890. La formación de la conciencia de clase y el giro emocional", Historiografías. Revista de historia y teoría, 10, (2015), pp. 31-48.

----- "Emociones y liderazgo político en la Vizcaya finisecular. Facundo Perezagua, la fuerza emocional del líder", Sancho el Sabio, 39, (2016), pp. 67-90.

---- Emociones obreras, política socialista. Movimiento obrero vizcaíno, 1886-1915, Madrid, Tecnos, 2018.

LAZARUS, Robert, "Thoughts on the relations between emotion and cognition", American Psychologist, 37, (1982), pp. 1019-1024. 
MATT, Susan y STEARNS, Peter (eds), Doing emotions history, Chicago, University of Illinois Press, 2014.

MIRALLES, Ricardo, "La gran huelga minera de 1890: en el origen del movimiento obrero en el País Vasco", Historia Contemporánea, 3, (1990), pp. 15-44.

NOVO LÓPEZ, Pedro, "El agua potable a domicilio y los residuos urbanos bailan un complejo pas a deux (1850-1930): una aproximación metodológica e historiográfica”, Historia contemporánea, 24, (2002), pp. 281-322.

NYE, Robert, Masculinity and male codes of Honor un Modern France, Berkeley, Berkeley University Press, 1993.

PÉREZ LEDESMA, Manuel, "El miedo de los acomodados y la moral de los obreros", en FOLGUERA, Pilar, Otras visiones de España, Madrid, Editorial Pablo Iglesias, 1993, pp. 27-64.

RALLE, Michel, "La sociabilidad obrera en la sociedad de la Restauración (1875-1910)", Estudios de Historia Social, Volumen 50/51, (1989), pp. 161-199.

---- "Las huelgas antes y después del $1^{\circ}$ de mayo", Estudios de Historia Social, vols 54/55, (1991), pp. 7-135.

REDDY, William M., The Navigation of Feeling. A framework for the history of Emotions, Nueva York, Cambridge University Press, 2001.

----- "Emotional styles and Modern forms of life" en KARAFYLLIS, Nicole, y ULSHOFER, Gotlind (eds.), Sexualized Brains: Scientific modeling of emotional intelligence from a cultural perspective, Cambridge, Cambridge University Press, 2008.

ROSALDO, Michelle, Knowledge and passion. Ilognot notions of self and social life, Cambridge, Cambridge University press, 1980.

RUZAFA, Rafael, Antes de la clase: los trabajadores de Bilbao y la margen izquierda del Nervión, 1841-1891, Leioa, Universidad del País Vasco, 1998.

SABORIT, Andrés, "Semblanza de Indalecio Prieto" El Socialista, 30 de abril 1953, pp. 3-4.

SCOTT, Joan W, “On language, gender and working-class history”, International Labor and Working-Class History, 31, (1987), pp. 1-13.

SERRANO, Carlos, "Cultura popular/cultura obrera en España alrededor de 1900", Estudios de historia social, Volumen 4, (1989), pp. 21-32.

SIERRA ÁLVAREZ, José, El obrero soñado. Ensayo sobre el paternalismo industrial (Asturias 1860-1917), Madrid, Siglo XXI, 1990.

----- "Rough Characters": Mineros, alcohol y violencia en el Linares de finales del siglo XIX", Historia Social, 19, (1994), pp. 77-98.

STEDMAN JONES, Gareth, Languages of Class: Studies in English working-class history, 1832-1982, New York, Cambridge University Press, 1983.

THOMPSON, Edward Palmer, The making of the English Working Class, New York, Pantheon Books, 1964 (1963).

---- "The moral economy of the English crowd in the Eighteenth century", Past and Present, (1971), pp. 76-136.

----- Miseria de la teoría, Barcelona, Crítica, 1981.

VALDOUR, James, El obrero español, experiencias vividas (País Vasco), Bilbao, Universidad del País Vasco, 2000 (1919).

VERGARA GARCÍA, Eugenio, Datos para la topografía médica de san Salvador del Valle, Impr. Bonifacio Guzmán, Baracaldo, 1904. 